\title{
Evanescent magnetic field effects on entropy generation at the onset of natural convection
}

\author{
MOURAD MAGHERBI ${ }^{a}{ }^{*}$, ATEF EL JERY $^{b}$, NEJIB HIDOURI $^{b}$, \\ AMMAR BEN BRAHIM ${ }^{b}$ \\ ${ }^{a}$ High Institute of Applied Sciences and Technology of Gabes, \\ Omar Ibn El Khattab Street, Gabes 6029, Tunisia \\ ${ }^{b}$ National School of Engineers of Gabes, Omar Ibn El Khattab Street, \\ 6029 Gabes, Tunisia \\ e-mail: magherbim@yahoo.fr; atefeljery@ @otmail.fr; n_hidouri@yahoo.com; \\ ammar.benbrahim@enig.rnu.tn
}

MS received 29 November 2008; revised 14 October 2009; accepted 20 October 2010

\begin{abstract}
This paper numerically investigates the effect of an externally evanescent magnetic field on total entropy generation in a fluid enclosed in a square cavity by using a control volume finite element method to solve the conservation equations at Prandtl number of 0.71 . The values of relaxation time of the magnetic field are chosen, so that the Lorentz force acts only in the transient state of entropy generation in natural convection. The total entropy generation was calculated for, fixed value of irreversibility distribution ratio, different relaxation time varying from 0 to $1 / 5$ and Grashof number varying from $10^{4}$ to $10^{5}$. The effects of the Hartman number and the magnetic field inclination angle on the evolution of total entropy generation throughout the transient regime were investigated. Results show that the application of evanescent magnetic field not only suppresses the fluctuation of the total entropy generation in the transient state, but also reduces the gap for magnetic field relaxation time less than $1 / 10$.
\end{abstract}

Keywords. Heat transfer; convection; magnetic field; cavity; entropy generation.

\section{Introduction}

Entropy generation analyses have become a very useful tool for thermal designs of thermodynamic systems in order to decrease energy loss. Various sources of irreversibility are responsible for entropy generation such as heat and/or mass transfer, magnetic field effect, viscous dissipation effect, etc. Many studies have been published on entropy generation. Bejan (1982 \& 1996) illustrated that the flow parameter could be selected in order to minimize the irreversibility associated with a specific convective heat transfer processes. For a mixed convective flow, Mahmud et al (2003) gave a detailed analysis of the entropy generation nature

${ }^{*}$ For correspondence 
and sources of irreversibility in a vertical non-porous channel with transverse hydromagnetic effect. Haddad et al (2004) conducted a study on entropy generation through a single rectangular microchannel with constant heat flux boundary condition. They modelled microchannel numerically in order to find velocity and temperature distributions along the coolant flow and channel width directions. Mahmud \& Fraser (2002) studied the entropy generation minimization concept for different geometric configurations, flow situations, and thermal boundary conditions. Furthermore, the influence of physical, geometrical and cost parameters on the optimum Reynolds numbers were presented by Shuja et al (1999) and Shuja (2002). In other study, Yilbas (2001) determined the heat transfer characteristics and resulting entropy generation across annuli with a rotating outer cylinder in linear velocity distribution. Entropy generation due to laminar mixed convection from an isothermal rotating cylinder was calculated numerically by Abu-Hijleh \& Helen (1999). Tasnim et al (2002) gave a detailed analysis of entropy generation and the source of irreversibility in a vertical porous media with transverse hydromagnetic effect for a mixed convective flow. Mahmud \& Fraser (2004) analysed the problem of entropy generation in a fluid-saturated porous cavity for laminar magnetohydrodynamic natural convection heat transfer. In a similar study, Mahmud \& Fraser (2003) investigated first law and second law aspects of fluid flow and heat transfer inside a vertical porous channel with a transverse magnetic field. Al-Odat et al (2004) investigated the magnetic field effect on local entropy generation in a steady two-dimensional laminar forcedconvection flow past a horizontal plate. Despite many studies of flow and heat field problems for various types of geometries, Baytas (1997) and Ogulato et al (1997) have not considered the effect of slip on entropy generation. Gad-el Hak (1999) showed that the velocity on the surface, where the effect of slip dominates, is related to the slip factor by the boundary conditions. Slip-dependent velocity means the reduction in loss of mechanical energy into thermal energy (i.e. reduction in entropy generation) results in more available energy. Also, relating entropy generation to slip factor in flow and heat field solutions gives more accurate results, which help the designer to produce correct efficiency calculations and geometrical optimization of a rotating system. De Groot \& Mazur (1984) studied the optimization criterion which is the minimization of the entropy generation rate. In the context of the classical thermodynamics of irreversible processes stationary non-equilibrium states are characterized by a minimum of the entropy production, compatible with the external constraints imposed on the system, provided that phenomenological coefficients are assumed constant. This ensures that the intrinsic irreversibility associated with a given physical process reaches a minimum consistent with the constraints demanded by the system. This optimization method has been applied in a number of heat transfer and fluid flow problems by Ibanez et al $(2002 ; 2003 \mathrm{a}$; 2003b) and 2004) as well as in flows of electrically conducting fluids under magnetic fields.

The present study is a parametric study and can be considered as extension of previous works in term of the choice for Prandtl number set to be $P r=0 \cdot 71$. Thus, Chaudhary \& Jain (2008) made analytical study of the transient hydromagnetic and thermal behaviour of free convection flow assuming an electrically conducting fluid. For physical meaning of the problem, values of Prandtl number are chosen as 0.71 (air), 1 (electrolytic solution) and 7 (water). It was found that the increase of Prandtl number induces the decrease of temperature. Magnetic field causes the decrease of velocity for both air and water. Teamah (2008) made numerical studies of steady heat and mass transfer by natural convection flow of a heat generating fluid in presence of a transverse magnetic field in a rectangular enclosure at fixed values of aspect ratio $(A=2)$. The study showed that the Lewis number $(L e=1)$ and Prandtl number $(\operatorname{Pr}=0 \cdot 7)$. It was found that magnetic field tends to reduce heat transfer and fluid circulation within the enclosure. For Hartman number $\mathrm{Ha}>20$, average Nusselt \& Sherwood 
numbers have constant values over a range of thermal Rayleigh number, this range increases with increasing Hartman number. Ishak et al (2008) made numerical studies of a steady twodimensional flow of an electrically conducting fluid due to a stretching of cylindrical tube. They showed that Nusselt number increases as Prandtl number increases (from $\mathrm{Pr}=0.7$ to $\mathrm{Pr}=7$ ). Moreover, the effect of magnetic field is found to be more pronounced for fluids with smaller Prandtl number $(P r=0 \cdot 7)$, since fluids with smaller Prandtl number have larger thermal diffusivity.

As a consequence and following the above works, the main objective of the present paper is to study the magnetic field effect on entropy generation evolution in transient heat transfer, at Prandtl number $0 \cdot 71$, without disturbing the steady state. This requires the use of an evanescent magnetic field, which has not yet been employed.

\section{Mathematical formulation}

Imposed evanescent magnetic field acting on Newtonian fluid enclosed in heated square cavity is considered in this problem (figure 1). The fluid is modelled as a Boussinesq incompressible fluid, whose properties are described by its kinematics viscosity, thermal diffusivity and thermal volumetric expansion coefficient. The induced magnetic field is negligible in comparison to the imposed field. The electric field must be irrotational $(\nabla \times E=0)$ and can be written as: $E=-\nabla \varphi$, where $\varphi$ is the electrostatic potential. The divergence of electric field leads to the following expression: $\nabla J=-\sigma_{e} \nabla^{2} \varphi+\sigma_{e} \nabla \cdot(w \times B)=0$, as it is given by Davidson (2001). It is assumed that there is no imposed electric field, therefore the electrostatic potential is equal to zero and the electric force is negligible as compared to the magnetic force $(w \times B)$ as given in Woods (1975). Consequently, the electric field is reduced to $J=\sigma_{e}(w \times B)$ and the magnetic force can be written as:

$$
F_{m}=J \times B=\sigma_{e}(w \times B) \times B .
$$

Under the above assumptions, the conservation equations for mass, momentum and energy in dimensionless form are as follows:

$$
\begin{aligned}
& \frac{\partial U}{\partial X}+\frac{\partial V}{\partial Y}=0 \\
& \frac{\partial U}{\partial \tau}+\operatorname{div}(U W-\operatorname{gradU})=-\frac{\partial P}{\partial X}+H a^{2}\left(V \sin \alpha \cos \alpha-U \sin ^{2} \alpha\right) \\
& \frac{\partial V}{\partial \tau}+\operatorname{div}(V W-\operatorname{gradV})=-\frac{\partial P}{\partial Y}+[G r \theta]+H a^{2}\left(U \cos \alpha \sin \alpha-V \cos ^{2} \alpha\right) \\
& \frac{\partial \theta}{\partial \tau}+\operatorname{div}\left(\theta W-\frac{1}{\operatorname{Pr}} \operatorname{grad} \theta\right)=0
\end{aligned}
$$

where the dimensionless variables are defined by:

$$
\begin{aligned}
X & =\frac{x}{L} ; \quad Y=\frac{y}{L} ; \quad U=\frac{u L}{v} ; \quad V=\frac{v L}{v} ; \quad \theta=\frac{T-T_{0}}{T_{h}-T_{c}} ; \quad P=\frac{p L^{2}}{\rho v^{2}} ; \\
G r & =\frac{g \beta\left(T_{h}-T_{c}\right) L^{3}}{v^{2}} ; \quad \tau=\frac{v t}{L^{2}} ; \quad H a^{2}=\frac{B^{2} L^{2} \sigma_{e}}{\mu} .
\end{aligned}
$$




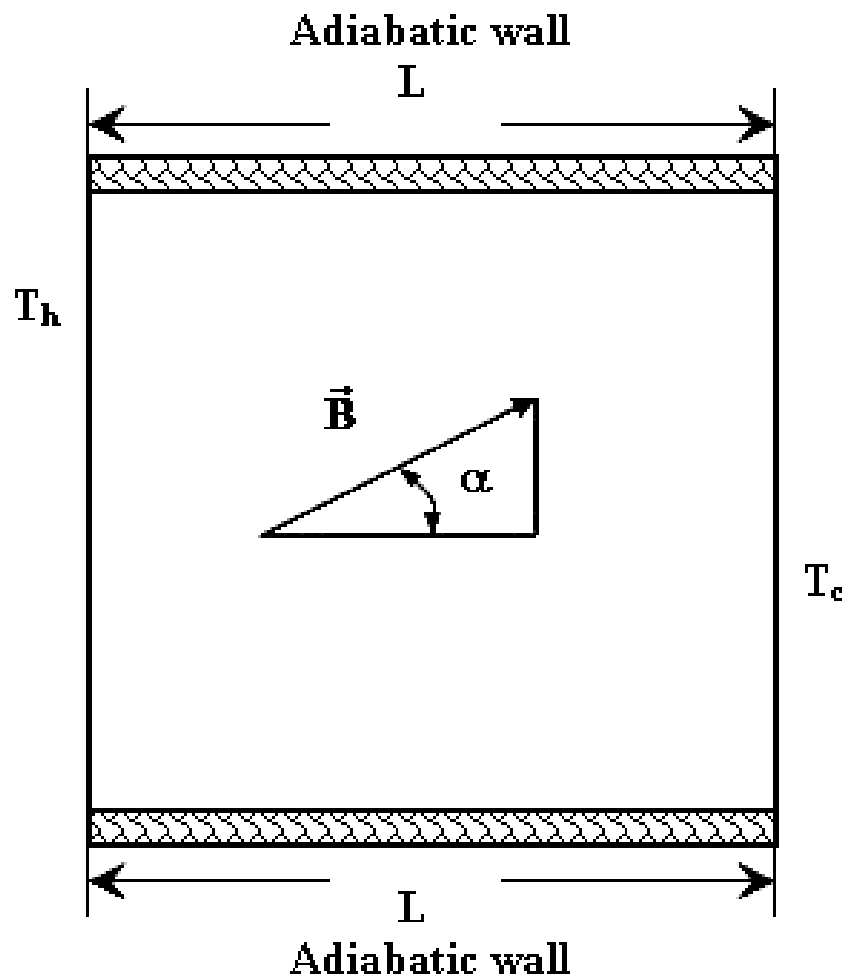

Figure 1. Schematic geometrical configuration of the problem under consideration.

The boundary conditions are:

$U=V=0$ for all walls; $\theta=0.5$ on plane $X=0$ and $\theta=-0.5$ on plane $X=1$

$\frac{\partial \theta}{\partial Y}=0$ on planes $Y=1$ and $Y=0$.

The initial conditions are:

At $\tau=0 ; U=V=P=0$ and $\theta=0.5-X$ for whole space.

\section{Entropy generation}

The irreversibility involved in the system due to momentum and energy transport between the vertical walls of the enclosure sets the fluid in non-equilibrium state and induces a continuous entropy production in the system. In two-dimensional coordinates system and using the dimensionless variables listed in equation (6), the dimensional local entropy generation was given by Woods (1975) and can be written in dimensionless form as:

$$
S_{l, a}=S_{l, a, H}+S_{l, a, F}+S_{l, a, M},
$$

with:

$$
S_{l, a, H}=\left(\frac{\partial \theta}{\partial X}\right)^{2}+\left(\frac{\partial \theta}{\partial Y}\right)^{2}
$$




$$
\begin{aligned}
S_{l, a, F} & =\chi_{1}\left[2\left(\frac{\partial U}{\partial X}\right)^{2}+2\left(\frac{\partial V}{\partial Y}\right)^{2}+\left(\frac{\partial U}{\partial Y}+\frac{\partial V}{\partial X}\right)^{2}\right] . \\
S_{l, a, M} & =\chi_{2}[U \sin \alpha-V \cos \alpha]^{2} .
\end{aligned}
$$

In the right hand side of equation (7), the first term represents the entropy generation due to heat transfer, the second is due to viscous dissipation while the third term is due to magnetic field.

Dimensionless terms $\chi_{i}(i=1 \cdot 2)$ are irreversibility distribution ratios given by:

$$
\chi_{1}=\frac{\mu T_{0}}{k}\left(\frac{v}{L\left(T_{h}-T_{c}\right)}\right)^{2} ; \quad \chi_{2}=\chi_{1} H a^{2} .
$$

The dimensionless total entropy generation over the system volume is defined by integrating equation (7):

$$
S_{t}=\int_{\Omega} S_{l, a} d \Omega
$$

\section{Numerical procedure}

A modified version of the control volume finite-element method (CVFEM) of Saabas \& Baliga (1994) is adapted to the standard staggered grids in which pressure and velocity components are stored at different points. The SIMPLER algorithm was applied to resolve the pressurevelocity coupling in conjunction with an alternating direction implicit (ADI) scheme for performing the time evolution. From the known temperature and velocity fields at any time $\tau$ given by solving equations (2-5), the local entropy generation $S_{l, a}$ is evaluated at any nodal point of the cavity by equation (7). The dimensionless total entropy generation for the entire cavity $S_{t}$ is easily obtained by equation (12). The shape function describing the variation of the dependant variables $\psi(=U, V, \theta)$ is needed to calculate the flux across the controlvolume faces. We have followed Saabas \& Baliga (1994) in assuming linear and exponential variations respectively when the dependant variable $\psi$ is calculated in the diffusive and in the convective terms of the conservation equations. The used numerical code written in FORTRAN language was described and validated in details as in Abbassi et al (2001a and b). More details and discussions about CVFEM are available in the works of Prakash (1986), Hookey (1989), Elkaim et al (1991) and Saabas \& Baliga (1994). Since entropy generation is a function of thermal and velocity gradients, it can be a good criterion for grid independence analysis. Figure 2 a illustrates the variation of total entropy generation values in the steady state versus grid size for different value of Grashof number. Results show that for $G r \leq 5 \times 10^{4}$, maximum relative error value in terms of entropy generation is equal to $1.53 \%$ when we pass from a grid size of $31 \times 31$ to $41 \times 41$ nodal points. For $G r=10^{5}$ the value of relative error is equal to $2.4 \%$ when we pass from a grid size of $31 \times 31$ to $41 \times 41$ nodal points and becomes $1.18 \%$ when we pass from a grid size of $41 \times 41$ to $51 \times 51$ nodal points.

In transient state, for $G r=10^{5}$ entropy generation and relative errors were calculated at $\tau=0.025, \alpha=0^{\circ}$ and $\mathrm{Ha}=0$ for different grids as is given in table 1 . When we pass from grid of $31 \times 31$ to $41 \times 41$ nodal points, relative error is equal to $1.48 \%$, this error becomes equal to $0.75 \%$ when we pass from $41 \times 41$ to $51 \times 51$. 

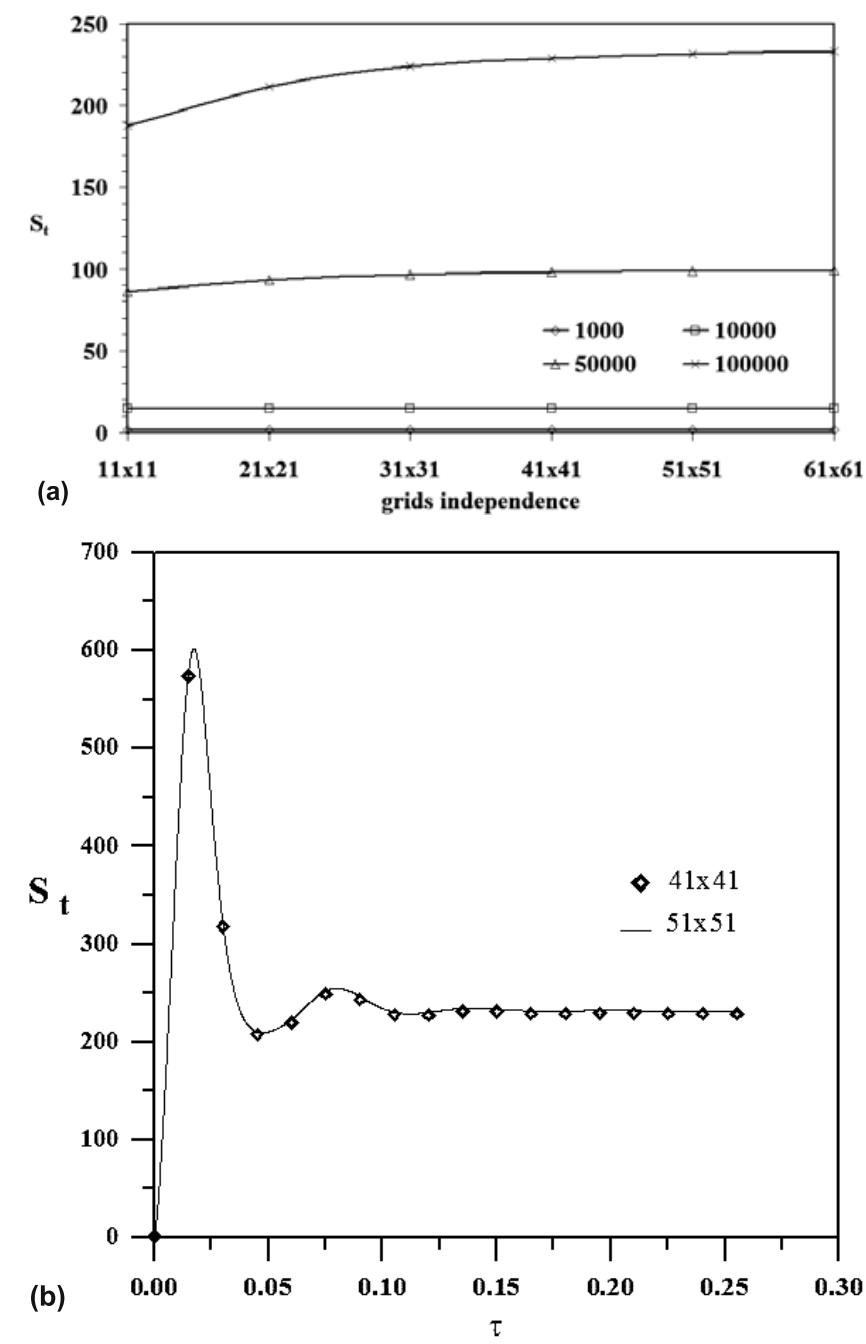

Figure 2. (a) Entropy production distribution for $\chi_{1}=10^{-3} \mathrm{Ha}=$ 0 and $\alpha=0^{\circ}$ all in function of grids. (b) Entropy generation versus time at $G r=10^{5}, \chi_{1}=$ $10^{-3} \mathrm{Ha}=0$ and $\alpha=0^{\circ}$ for $41 \times 41$ and $51 \times 51$ meshes.

Table 1. Transient entropy generation and relative error at: $\tau=0.025 ; H a=0 ; \alpha=0^{\circ} ; G r=10^{5}$.

\begin{tabular}{lcc}
\hline & \multicolumn{2}{c}{ Transient state } \\
\cline { 2 - 3 } Grid size & $S_{t}$ & Relative error \\
\hline $31 \times 31$ & 436,069 & \\
$41 \times 41$ & 442,503 & $1,48 \%$ \\
$51 \times 51$ & 445,843 & $0,75 \%$ \\
\hline
\end{tabular}

With relative error: $E R=\left|\frac{S_{t(i+1, i+1)}-S_{t(i, i)}}{S_{t(i, i)}}\right|$ 
From stationary and transient states, it's clear that the use of $31 \times 31,41 \times 41$ and $51 \times 51$ nodal points for $G r \leq 10^{4}, 5 \times 10^{4}$ and $10^{5}$ respectively is sufficiently enough to carry out this study. Further, figure $2 b$ shows the variation of transient entropy generation for $41 \times 41$ and $51 \times 51$ meshes at $G r=10^{5}$. As can be seen only a small increase of entropy generation magnitude is obtained. The transient study is carried out with a step time $\Delta \tau=10^{-4}$ for all considered Grashof numbers.

\section{Results and discussion}

All industrial systems induce entropy generation causing energy degradation and then reduction in efficiency. We are also convinced that a part of total entropy generation is at the origin of gradual deterioration of mechanical system (principally at the starting operation). Numerous investigations were carried out with an aim of optimizing entropy generation. In several real cases, we believe that optimizing entropy generation in the stationary state can often change the desired product characteristics, which is generally disagreeable. This requires an optimisation of the entropy generation only in transient state by applying an evanescent magnetic field (equation (13)) on the fluid enclosed in a square cavity. This can make profit of available work, minimize the potential damage at the beginning of the operation system and additionally maintain the characteristics of the end product. We believe that some applications of this study can hold turbo-machinery, pumps, compressors and internal combustion engine. The evanescent magnetic field can be written as:

$$
B=B_{0} e^{-\gamma t} \quad\left(\gamma \in I R^{+}\right) .
$$

Using equation (6), the Hartman number is a decreasing function versus time and can be written as:

$$
H a=H a_{0} e^{-\left(\frac{\gamma L^{2}}{v}\right) \tau} .
$$

The parameter $\gamma$ was selected so that the inverse of the magnetic field relaxation time $\frac{1}{\xi}=$ $\left(\frac{\gamma L^{2}}{v}\right)$ takes natural number. Therefore, the Hartman number can be written as:

$$
H a=H a_{0} e^{-\frac{\tau}{\xi}}
$$

In this study, the Prandlt number is equal to $0 \cdot 71$. The Grashof number and the inclination angle of the magnetic field are varying from $10^{4}$ to $10^{5}$ and from $0^{\circ}$ to $90^{\circ}$, respectively. The initial Hartman number $\left(H a_{0}\right)$ is equal to 100 . The irreversibility distribution ratio $\chi_{1}$ is kept constant and equal to $10^{-3}$. It is important to note that, for higher values of relaxation time $\xi$, the magnetic field takes practically constant value and can therefore disturb the stationary state. At primary, the inclination angle of the magnetic field is kept constant which is equal to zero. The values of parameter $\xi$ are chosen so that the magnetic field acts only in the transient state of natural convection. This is illustrated in figure 3 which shows the variation of the entropy generation and Hartman number versus time. It can be concluded from this figure that the dimensionless total entropy generation suddenly increases and takes maximum value $\left(S_{t, \text { Max }}\right)$ at the very beginning of natural convection. Thereafter, it decreases with oscillatory behaviour to finally reach a constant value in the steady state. Fluctuations of the total entropy generation for Grashof number equal to $10^{5}$ indicate that the flow exhibits 


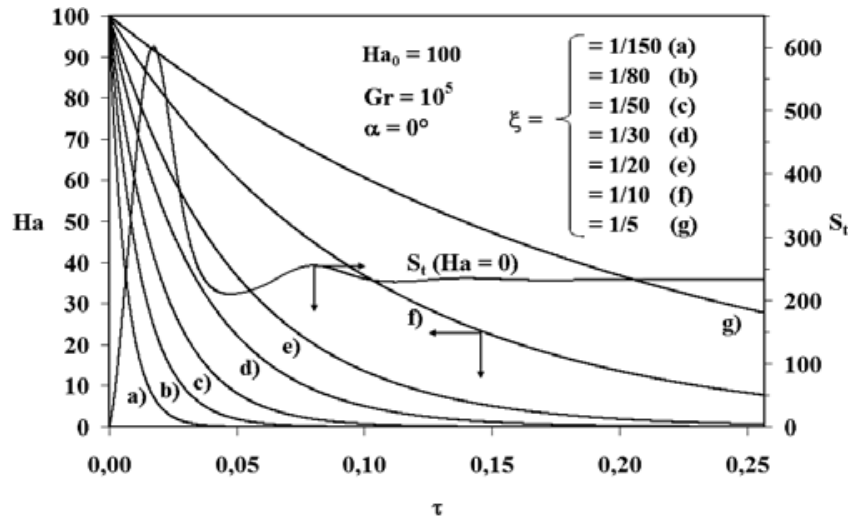

Figure 3. Hartman number for different relaxation times $\xi$ and entropy production distribution for $\chi_{1}=10^{-3} \mathrm{Ha}=0, \mathrm{Gr}=$ $10^{5}$ and $\alpha=0^{\circ}$ all in function of dimensionless time.

oscillatory behaviour which depends on the boundary conditions, as can be seen in figure 4 . It is important to note from this figure that the amplitude and the numbers of oscillations are as significant as the Grashof number (boundary conditions) is important. This result is consistent with the findings of Ivey (1984). Schladow (1990) and Magherbi et al (2003). The latter explained, from a thermodynamic viewpoint that, for Rayleigh number greater then the critical Rayleigh number $R a_{c}=5200$ (which corresponds for Prandtl number equal to 0.71 to a critical Grashof number equal to 3700 ), the steady state is relatively far from equilibrium state, then, a rotation around the steady state is possible and the system is in the case of a spiral approach towards this state corresponding to an oscillation of the total entropy generation. Consequently, the system evolves in the non-linear branch of irreversible phenomena, since the Prigogine's theorem of minimum entropy production is unproven. Let us consider the difference between the value of the maximum of entropy generation at the onset of natural convection and its value at the steady state denoted by Magherbi et al (2003) as the Gap $\left(G=S_{t, \text { Max }}-S_{t, s}\right)$. As can be seen from figure 3, the value of entropy generation at the onset of natural convection $\left(S_{t, \operatorname{Max}}\right)$ is practically twice larger than its value at the steady state. This involves an important Gap and as a result an important losses of system available work in the transient state of convective heat transfer. To overcome this problem, we applied an evanescent magnetic field with various relaxation time values. The latter was selected so that the magnetic field affects only the transient state. This choice limits the relaxation time variation domain

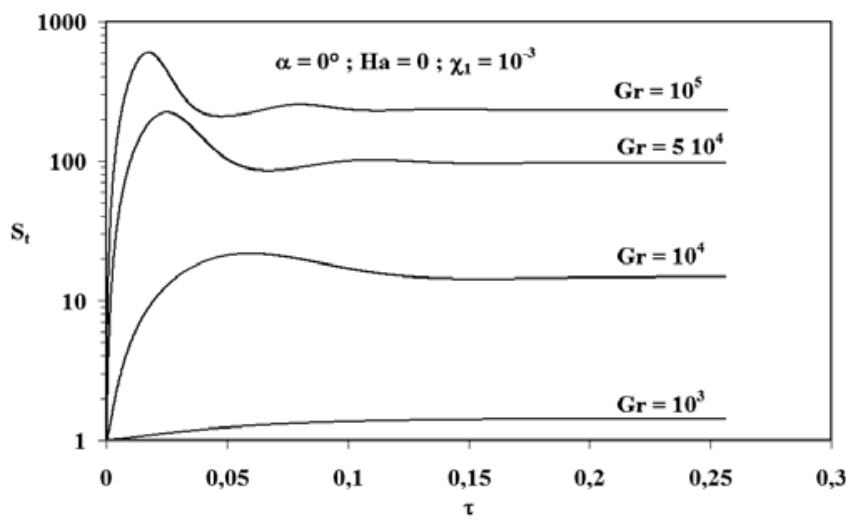

Figure 4. Entropy generation versus dimensionless time for different Grashof numbers at $\alpha=$ $0^{\circ}, H a=0$ and $\chi_{1}=10^{-3}$. 


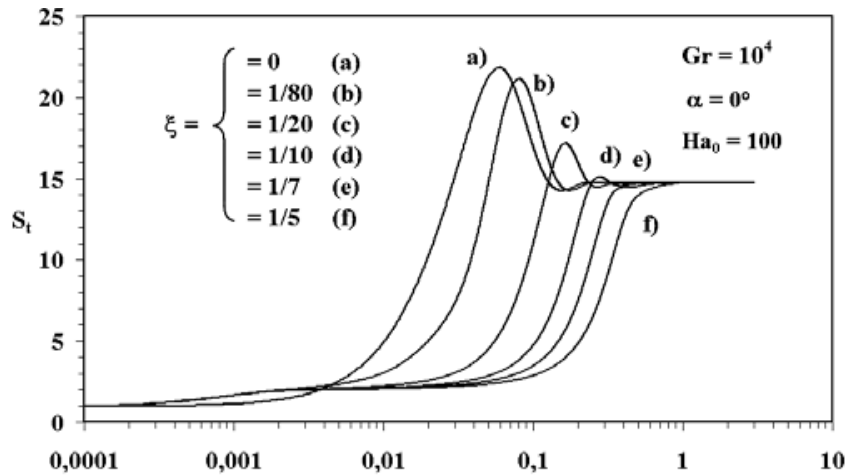

(a)

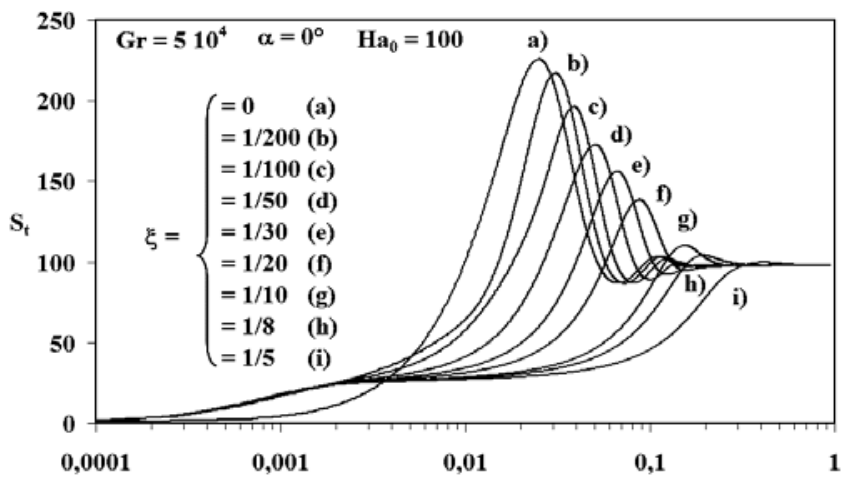

(b)

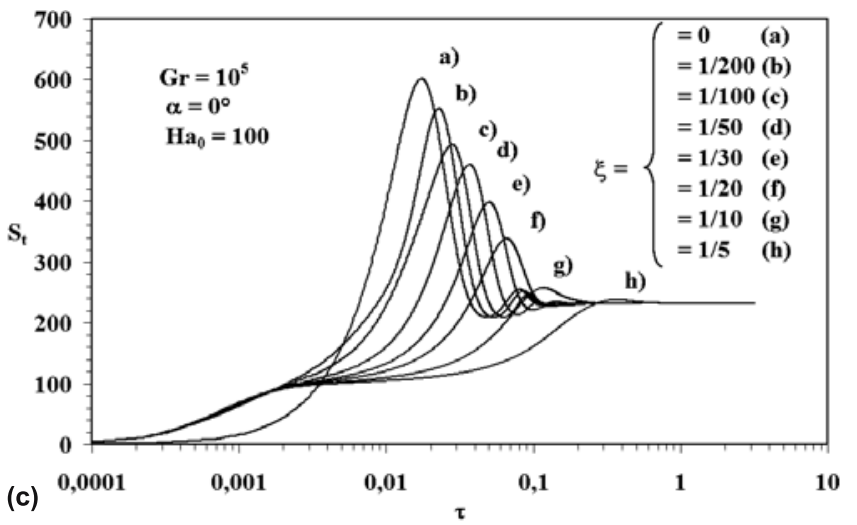

Figure 5. (a) Entropy generation versus dimensionless time for different relaxation time at $\alpha=$ $0^{\circ}, H a_{0}=100$ and $G r=10^{4}$. (b) Entropy generation versus dimensionless time for different relaxation time at $\alpha=0^{\circ}$, $H a_{0}=100$ and $G r=5 \times 10^{4}$. (c) Entropy generation versus dimensionless time for different relaxation time at $\alpha=0^{\circ}, H a_{0}=$ 100 and $G r=10^{5}$.

to be lower than $1 / 20$, as illustrated in figure 3 . The effect of Hartmann number on entropy generation fluctuations for different Grashof number is illustrated in figures 5(a-c) (the $X$ axis is given in logarithmic scale). From these figures, it is clear that, for fixed value of Grashof number entropy generation value at the steady state of natural convection is the same for all considered relaxation times. This result is very logical since the magnetic field takes zero value at the steady state. For example, at steady state and for Grashof number equal to $10^{5}$, the flow structure is the same as that obtained in the absence of magnetic field, which consists in a double spiral configuration and viscous boundary layers in close proximity to the 
active walls. Similar observations regarding the evolution of the dimensionless total entropy generation to the ones given in figure 3 can be conducted from figures $5(\mathrm{a}-\mathrm{c})$, except the influence of the relaxation time on the magnitude and the number of oscillations of total entropy generation. As can be seen from the previous figures, for a given Grashof number, total entropy generation begins to increase at the very start of the transient state due to the initial conditions of the fluid temperature and velocity. This augmentation is more significant as the Grashof number increases. It's important to notice that, at fixed relaxation time, the maximum and the number of oscillations of entropy generation are as important as the Grashof number is higher. No significant influence of the relaxation time (except for zero magnetic field) on the dimensionless total entropy generation was seen for dimensionless time around $210^{-3}$ for all considered Grashof numbers, since curves of entropy generation practically coincide. This is because the entropy generation at the very beginning of transient state is mainly due to the relatively important Hartman number and, therefore, magnetic irreversibly. As time proceeds, a bifurcation of the total entropy generation depending on the values of the relaxation time was observed. From the bifurcation point, as shown in figures $5(\mathrm{a}-\mathrm{c})$ and for fixed Grashof number, as the relaxation time increases, maximum of entropy generation magnitude decreases and occurs at longer time from the beginning of the transient state. Also, the number and the amplitude of oscillations of the total entropy generation decrease. This can be explained by the fact that for relatively higher Grashof number, the internal thermal and viscous waves resulting from the transition from one to double configuration in the whole cavity are gradually suppressed by the Lorentz force when the relaxation time increases. No oscillations are observed at critical relaxation time equal to $1 / 7,1 / 8$ and $1 / 10$ for Grashof number equal to $10^{4} .5 \cdot 10^{4}$ and $10^{5}$ respectively. Accordingly, the total entropy generation tends asymptotically towards the steady state, this is due to the adequate value of the Hartmann number throughout the transient regime and precisely when the transition to the double configuration occurs, for $G r \geq 510^{4}$. So, the Lorentz force is always able to eliminate the internal thermal and viscous waves. From a thermodynamic viewpoint, for relaxation time $\xi \geq 1 / 10$ according to Grashof number equal to $10^{5}$, the asymptotic behaviour of the total entropy generation shows that the system returns directly towards the steady state. Therefore, the Prigogine's theorem of minimum entropy production is verified. This can allow us to extend the concept of close equilibrium states by introducing new coupling effects due to the magnetic field action, and thereafter, enhance the validity domain of the linear branch of the thermodynamics of irreversible processes. Figure 6 illustrates the variation of the gap versus the relaxation time for different Grashof numbers. Results show that, for low fixed value of relaxation time, the gap increases with Grashof number. This is due to the fact that the system is as far from equilibrium state as the Grashof number increases. Consequently, the maximum of entropy generation reached at the very beginning of the transient state takes high magnitude compared to its value in the steady state. It can be seen from this figure that for fixed Grashof number, the Gap decreases as the relaxation time increases and becomes zero for higher values of relaxation time. The decrease of the Gap is more significant for relaxation time less than $1 / 10$ and is as important as the Grashof number. In fact, the magnetic field seems to suppress convection and to retard fluid motion via the Lorentz force. Therefore, the velocity and temperature gradients diminish inducing a decrease of the entropy generation in the cavity. It is important to notice that the Gap becomes insignificant and takes linear behaviour at higher values of relaxation time (figure 6). This is due to the fact that the duration and the intensity of the magnetic field and consequently the Lorentz force actions is as significant as the relaxation time, bringing an important decrease of the entropy generation throughout the transient state of natural convection. The influence of the magnetic field inclination angle 


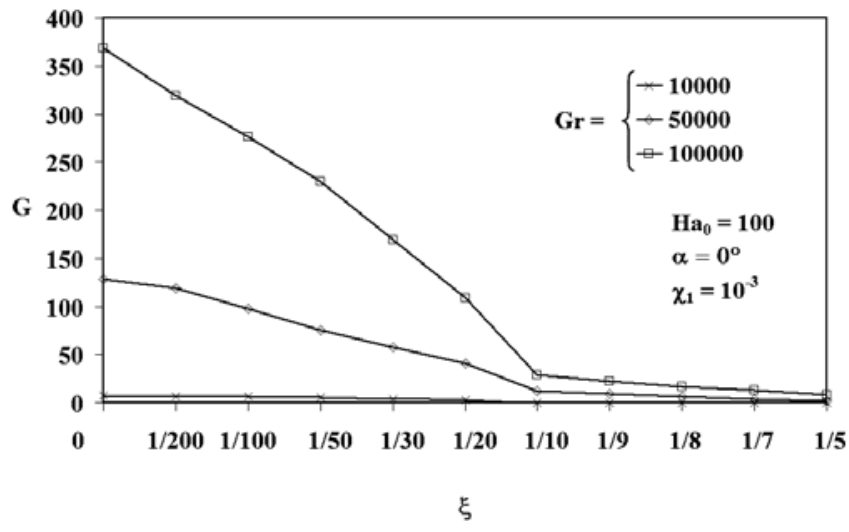

Figure 6. Gap versus relaxation time for different Grashof numbers at $\alpha=0^{\circ}, H a_{0}=100$ and $\chi_{1}=10^{-3}$.

on the total entropy generation is also studied for relaxation time equal to $1 / 5$ and $1 / 20$ and for inclination angle equal to $0^{\circ}, 30^{\circ}, 60^{\circ}$ and $90^{\circ}$. The Grashof number and irreversibility distribution ratio are considered constants and equal to $10^{5}$ and $10^{-3}$ respectively. Figure 7 shows that the magnetic field inclination angle acts only during the transient state, because of the zero intensity of the magnetic field in the steady state. It can be seen from this figure that the value and the dimensionless time corresponding to the maximum of entropy generation is affected by the inclination angle of magnetic field. For example, at relaxation time 1/20 the maximum of entropy generation takes minimum value at $30^{\circ}$ and maximum value at $60^{\circ}$.

\section{Conclusion}

Imposed evanescent magnetic field acting on Newtonian Boussinesq incompressible fluid enclosed in heated square cavity was investigated. The conclusions are as follows:

- The relaxation time should be selected so that the magnetic field acts in transient state only.

- Amplitude and oscillation numbers of entropy generation are important as Grashof number increases for any fixed relaxation time.

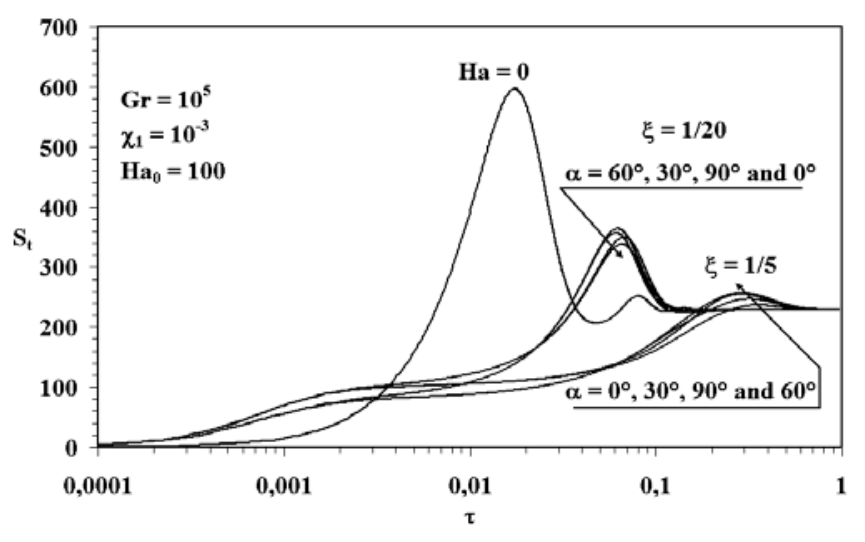

Figure 7. Entropy generation versus dimensionless time for different inclination angles of magnetic field and relaxation times at $G r=10^{5}, H a_{0}=100$ and $\chi_{1}=10^{-3}$. 
- The increase of relaxation time induces the decrease of maximum entropy generation value that occurs at longer time from the beginning of the transient state.

- Critical relaxation time from which no oscillations of transient entropy generation in natural convection are obtained and are: $1 / 7,1 / 8$ and $1 / 10$ for $G r=10^{4}, 510^{4}$ and $10^{5}$, respectively.

- Maximum transient entropy generation is obtained at $\alpha=60^{\circ}$, and the inclination angle of magnetic field induces a small shift of this maximum.

Authors would like to acknowledge the reviewers for their useful suggestions.

\section{Nomenclature}

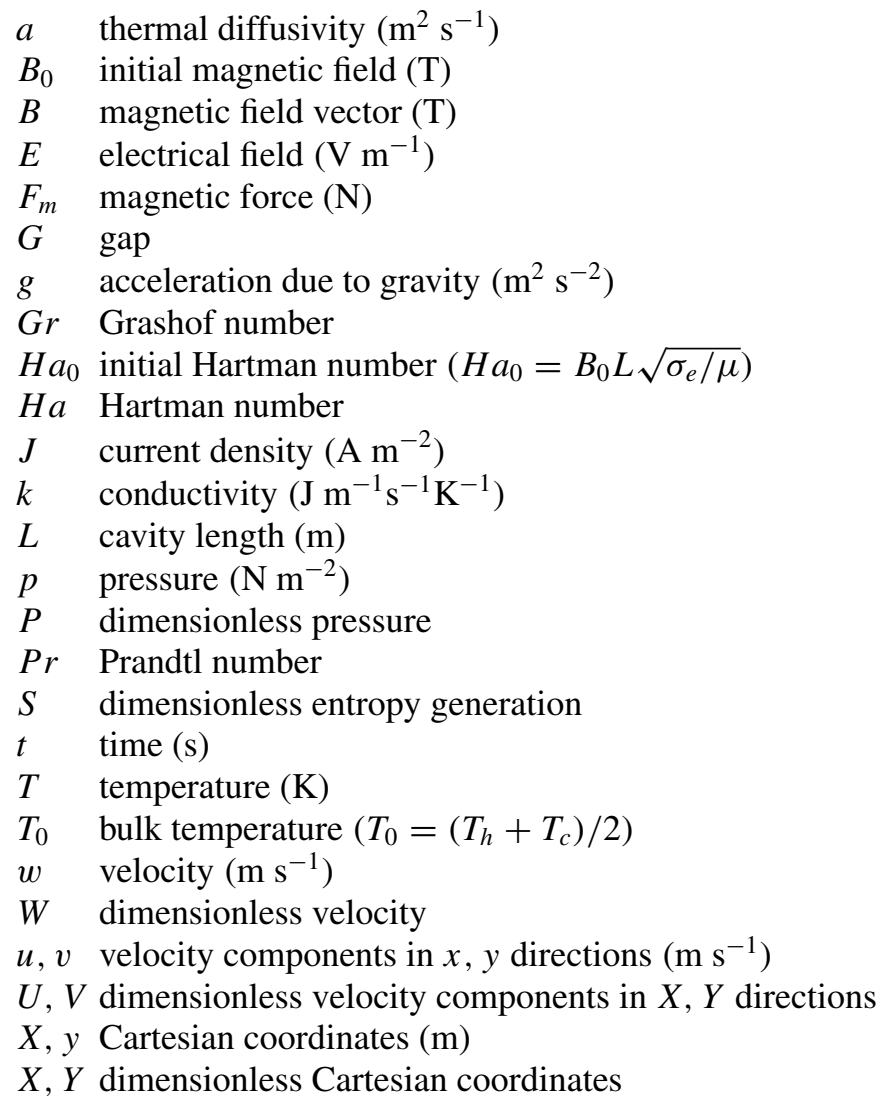

\section{Greek symbols}

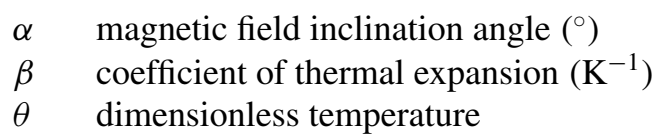




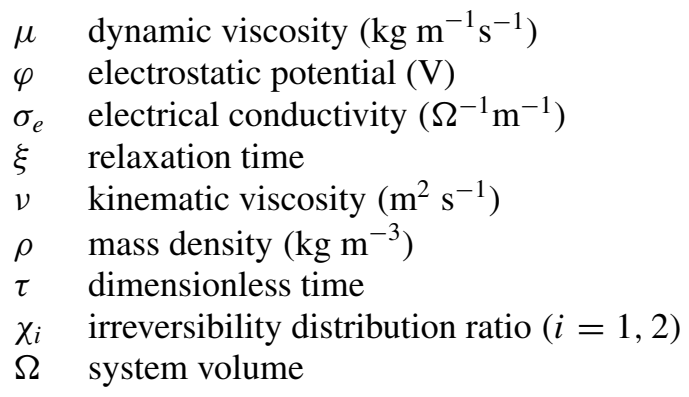

\section{Subscripts}

$\begin{array}{ll}a & \text { dimensionless } \\ c & \text { cold wall } \\ F & \text { fluid friction } \\ H & \text { heat } \\ h & \text { hot wall } \\ l & \text { local } \\ M & \text { magnetic } \\ \text { Max maximum } \\ t & \text { total } \\ s & \text { stationary }\end{array}$

\section{References}

Abbassi H, Turki S, Ben Nasrallah S 2001a Mixed convection in a plane channel with a built-in triangular prism. Numerical Heat Transfer (part A) 39(3): 307-320

Abbassi H, Turki S, Ben Nasrallah S 2001b Numerical investigation of forced convection in a plane channel with a built-in triangular prism. Int. J. Thermal Sciences 40: 649-658

Abu-Hijleh B A K, Helen W N 1999 Entropy generation due to laminar natural convection over a heated rotating cylinder. Int. J. Heat Mass Transfer 42: 4225-4233

Al-Odat M Q, Damseh R A, Al-Nimr M A 2004 Effect of magnetic field on entropy generation due to laminar forced convection past a horizontal flat plate. Entropy 4(3): 293-303

Baytas A C 1997 Optimization in an inclined enclosure for minimum entropy generation in natural convection. J. Non-Equil. Thermodyn. 22(2): 145-55

Bejan A 1982 Second-law analysis in heat transfer and thermal design. Advanced Heat Transfer 15: $1-58$

Bejan A 1996 Entropy Generation Minimization (New York: CRC Press)

De Groot S R, Mazur P 1984 Non-Equilibrium Thermodynamics (New York: Dover)

Chaudhary R C, Jain A 2008 Magnetohydrodynamic transient convection flow past a vertical surface embedded in a porous medium with oscillating temperature. Turkish J. Eng. Env. Sci. 32: 13-22

Davidson P A 2001 An Introduction to Magnetohydrodynamics. Cambridge University: Press, Cambridge

Elkaim D, Reggio M, Camarero R 1991 Numerical solution of reactive laminar flow by a controlvolume based finite-element method and the vorticity-stream function formulation. Numerical Heat Transfer (part B) 20: 223-240

Gad-el Hak M 1999 The fluid mechanics of microdevices - the Freeman Scholar lecture. J. Fluid Eng. ASME 121(1): 5-33 
Haddad O, Abuzaid M, Al-Nimr M 2004 Entropy generation due to laminar incompressible forced convection flow through parallelplates microchannel. Entropy 6(5): 413-426

Hookey N A 1989 A CVFEM for two-dimensional viscous compressible fluid flow. Ph.D. thesis; (Montreal Quebec: McGill University)

Ibáñez G, Cuevas S, López de Haro M 2002 Optimization analysis of an alternate magnetohydrodynamic generator. Energy Conversion and Management 43: 1757-1771

Ibáñez G, Cuevas S, López de Haro M 2003a Minimization of entropy generation by asymmetric convective cooling. Int. J. Heat Mass Transfer 46: 1321-1328

Ibáñez G, Cuevas S, López de Haro M 2003b Heat transfer in asymmetric convective cooling and optimized entropy generation rate. Rev. Mex. Fís. 49(2): 338-343

Ibáñez G, López de Haro M, Cuevas S 2004 Thermodynamic optimization of radial MHD flow between parallel circular disks. J. Non-Equil. Thermodynamics 29: 107-122

Ishak A, Nazar R, Pop I 2008 Magnetohydrodynamic (MHD) flow and heat transfer due to a stretching cylinder. Energy Conversion and Management 49: 3265-3269

Ivey G N 1984 Experiments on transient natural convection in a cavity. J. Fluid Mechanics 144: 389-401

Maghrebi M, Abbassi H, Ben Brahim A 2003 Entropy generation at the onset natural convection. Int. J. Heat and Mass Transfer 46: 3441-3450

Mahmud S, Fraser R A 2002 Second law analysis of heat transfer and fluid flow inside a cylindrical annular space. Exergy 2: 322-329

Mahmud S, Fraser R A 2003 Mixed convection-radiation interaction in a vertical porous channel: Entropy generation. Energy 28: 1557-1577

Mahmud S, Tasnim S H, Mamun M A H 2003 Thermodynamic analysis of mixed convection in a channel with transverse hydromagnetic effect. Int. J. Therm. Sci. 42: 731-740

Mahmud S, Fraser R A 2004 Magnetohydrodynamic free convection and entropy generation in a square porous cavity. Int. J. Heat Mass Transfer 47: 3245-3256

Ogulata R T, Doba F, Yilmaz T 1997 Second-law and experimental analysis of a cross-flow heat exchanger. Heat Transfer Eng. 20(2): 20-27

Prakash C 1986 An improved control volume finite-element method for heat and mass transfer, and for fluid flow using equal order velocity-pressure interpolation. Numerical Heat Transfer, Part B: Fundamentals 9: 253-276

Saabas H J, Baliga B R 1994 Co-located equal-order control-volume finite-element method for multidimensional, incompressible, fluid flow part I: formulation. Numerical Heat Transfer (part B) 26: 381-407

Schladow S G 1990 Oscillatory motion in a side-heated cavity. J. Fluid Mech. 213: 589-610

Shuja S Z, Zubair S M, Khan M S 1999 Thermoeconomic design and analysis of constant crosssectional area fins. Heat and Mass Transfer 34: 357-364

Shuja S Z 2002 Optimal fin geometry based on exergoeconomic analysis for a pin-fin array with application to electronic cooling. Exergy 2: 248-258

Tasnim S H, Mahmud S, Mamun M A H. 2002 Entropy generation in a porous channel with hydromagnetic effect. Exergy 2: 300-308

Teamah M A 2008 Numerical simulation of double diffusive natural convection in rectangular enclosure in the presences of magnetic field and heat source. Int. J. Thermal Sciences 47: 237-248

Woods L C 1975 The thermodynamics of fluid systems (Oxford Press: Oxford University)

Yilbas B S 2001 Entropy analysis of concentric annuli with rotating outer cylinder. Exergy 1(1): 60-66 